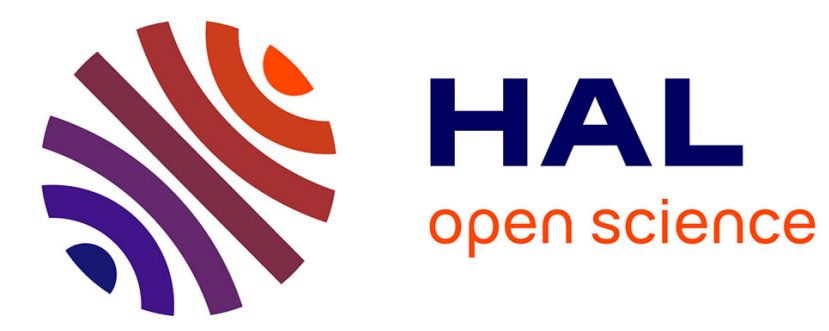

\title{
Domain Freezing in Triglycine Sulphate
}

Yue Huang, Xiaojian Li, Y. Ding, H. Shen, Zhen Zhang, Y. Wang, C. Fang, S. Zhuo

\section{To cite this version:}

Yue Huang, Xiaojian Li, Y. Ding, H. Shen, Zhen Zhang, et al.. Domain Freezing in Triglycine Sulphate. Journal de Physique IV Proceedings, 1996, 06 (C8), pp.C8-815-C8-818. 10.1051/jp4:19968175 jpa00254611

\section{HAL Id: jpa-00254611 https://hal.science/jpa-00254611}

Submitted on 1 Jan 1996

HAL is a multi-disciplinary open access archive for the deposit and dissemination of scientific research documents, whether they are published or not. The documents may come from teaching and research institutions in France or abroad, or from public or private research centers.
L'archive ouverte pluridisciplinaire HAL, est destinée au dépôt et à la diffusion de documents scientifiques de niveau recherche, publiés ou non, émanant des établissements d'enseignement et de recherche français ou étrangers, des laboratoires publics ou privés. 


\title{
Domain Freezing in Triglycine Sulphate
}

\author{
Y.N. Huang, X. Li, Y. Ding, H.M. Shen, Z.F. Zhang, Y.N. Wang, C.S. Fang* and S.H. Zhuo* \\ National Laboratory of Solid State Microstructures, Nanjing University, Nanjing 210093, China \\ * National Laboratory of Crystal Growth, Sandong University, Jinan, 250100, China
}

\begin{abstract}
Domain freezing is observed for the first time in triglycine sulphate. A new domain freezing model is proposed based upon the collective-pinning of random distributing pinning centers to DW's, and Vogel-Fulcher relation, which describes the relaxation during the process of domain freezing successfully, is deduced.
\end{abstract}

\section{INTRODUCTION}

Domain freezing is a process that domain walls (DW's) translate from movable (high temperature) to unmovable (low temperature) at finite temperature $T_{f}$. As firstly discussed by Barkla and Finlayzon [1] in 1953, the domain freezing phenomenon has been known for a long time and was intensively studied in recent years. At least three different and representative models have been put forward to describe this phenomenon: (i) Bornarel[2] proposed a model in 1972: Domain freezing is correlated to the critical shear stress necessary to move the quasidislocations of edge type in DW's. (ii). Another model proposed by Fedsov and Sidorkin[3] in 1977: There are two configurations of $D W$ 's and $T_{f}$ corresponds to the temperature where the two DW configurations inverse themselves. This induces modifications in the lattice energy barrier and then in the wall mobility. (iii) Kuramoto[4] proposed a model in 1987: Relaxation time $\tau$ of dipole reversal in DW's increases remarkably as temperature decreases and becomes infinite at certain temperature $\left(T_{f}\right)$, where the configuration entropy reaches a limiting value for the dipole reversal of two dimensional clusters. At the same time, thermal motion of DW's diminishes because of the abrupt increase of $\tau$ of dipole reversal in DW's, i.e. domain freezing.

Although above models can explain some experiential results, some questions are still open. For example, when measurement external field goes to zero, $T_{f}$ tends to $T_{C}$ according to Bornarel's model[2], but this is not consistent with the experimental results of Nakamura and Kuramoto[5]. As Bornarel pointed out[6], it seems difficult to imagine that Fedsov and Sidorkin's model with only short-range interaction being taken into account can explain the dependence of $T_{f}$ versus the sample thickness $d$. At the same way, this effect is not considered by Kuramoto[4]. In $\mathrm{KDP}$ doped with $\mathrm{KOH}$, $\mathrm{T}_{\mathrm{C}}$ is the same as that of ordinary KDP, which implies that the parameter for proton tunneling does not change, while $T_{f}$ decreases with increasing defect density. Nakamura and Kuramoto[5] stressed this fact means that, the domain freezing in KDP family is not phase transition with $\mathrm{T}_{\mathrm{f}}$ only dependent on the parameter for proton tunneling suggested by Fedsov and Sidorkin[3]. In short; as Kubinec et al.[7] pointed out (1995): "although the domain freezing phenomenon has been known for a long time and was intensively studied in recent years, the problem has not been solved yet." In the present paper, a new domain freezing model is proposed based upon the collective-pinning of pinning centers to DW's, and Vogel-Fulcher relation, which describes the 
relaxation during the process of domain freezing successfully, is deduced.

Up to now, domain freezing is only observed in crystals of KDP family[1-7], whether does it exist in other crystals, such as standard ferroelectrics triglycine sulphate (TGS)? One part of the present paper is that complex dielectric costant $\varepsilon=\varepsilon^{\prime}-1 \varepsilon$ " related to DW's is studied in TGS, and domain freezing is

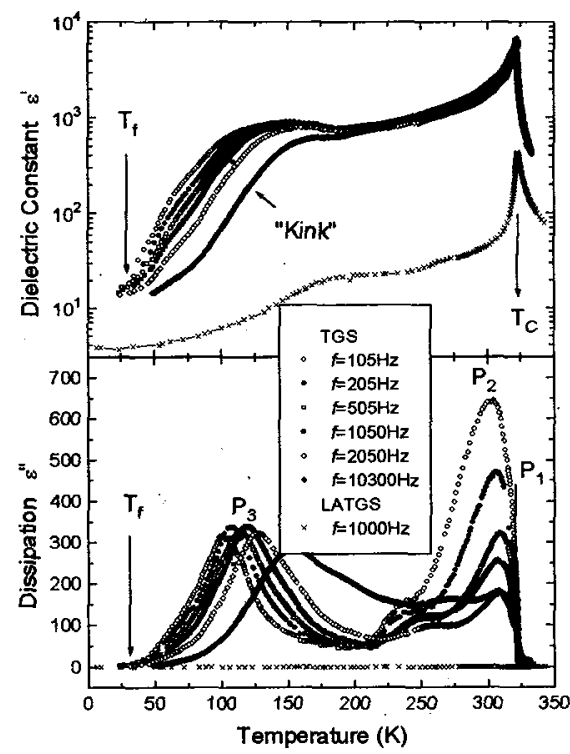

Fig. 1 The dependence of $\varepsilon$ 'and $\varepsilon$ " in TSS and LATGS versus $T$ at different measuring $f$.

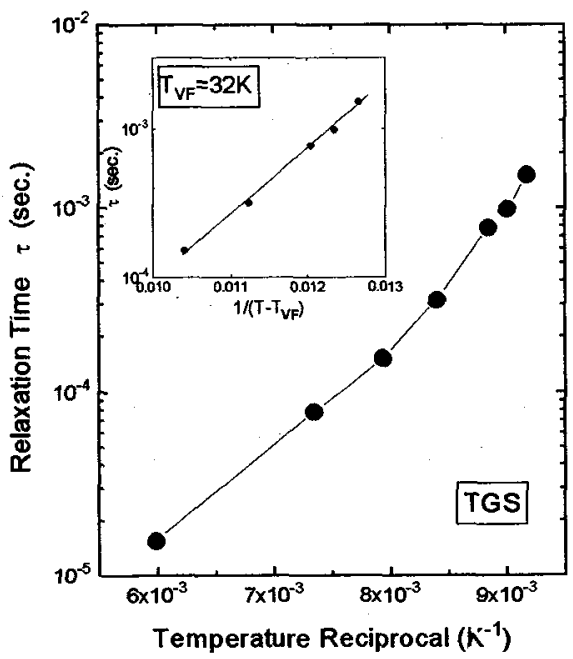

Fig. 2 Relaxation time $\tau$ vs $\mathrm{T}$ reciprocal in TGS. Inset: $\tau$ vs $\left(T-T_{\mathrm{VF}}\right)^{-1}$ observed for the first time.

\section{EXPERIMENTAL}

Triglycine sulfate $\left(\mathrm{NH}_{2} \mathrm{CH}_{2} \mathrm{COOH}\right)_{3} \mathrm{H}_{2} \mathrm{SO}_{4}$ (TGS), triglycine sulfate doped with $\alpha$-alanine (LATGS) single crystals were obtained from the aqueous solution by slow evaporation. Ferro-paraelectric transitions of second order take place at $322 \mathrm{~K}$ and $323 \mathrm{~K}$ in TGS and LATGS, respectively. There exist poly-domains $\left(180^{\circ}\right)$ in ferroelectric phase in TGS. Doping $\alpha$-alanine in TGS (LATGS) induces internal electric field which polarizes crystals to mono-domain state, but its influence on the Curie temperature $T_{C}$ is little, $T_{C}$ shifts to higher temperature only $\sim 1 \mathrm{~K}[8]$. All TGS and LATGS samples are prepared as platelets with size $a \times b \times c=5 \times 0.6 \times 5 \mathrm{~mm}^{3}$, and each $b$-surface evaporated with silver.

The complex dielectric constant $\varepsilon=\varepsilon^{\prime}-\mathrm{i} \varepsilon$ " was measured using a GA-1615A capacitance electric bridge from $10 \mathrm{~Hz}$ to $100 \mathrm{kHz}$. The method of scanning temperature $T$ at a fixed frequency $f$ in a cooling and heating circles is used and $f$ is changed in a following circle. The scanning rate is $\sim 1 \mathrm{~K} / \mathrm{min}$.

\section{RESULTS}

As shown in Fig. 1, there is a $\lambda$-shape peak of $\varepsilon$ ' just at $T_{C}$, and between a certain temperature internal below $\mathrm{T}_{C}, \varepsilon$ ' exhibits anomalous high value ("plateau" region) as compared to that predicted by the phenomenological Landau theory (about two orders). Then at a certain temperature $\varepsilon$ ' abruptly falls down to its phenomenological value. This feature looks like a "kink". In KDP family[1-7], the "plateau" region of $\varepsilon$ ' is believed to be due to the motion of $D W$ 's, and the abrupt decrease of $\varepsilon^{\prime}$ is attributed to domain freezing and this point is defined as a freezing temperature $T_{f}$. Through the comparison of the results of $\varepsilon$ ' between TGS and LATGS, and $\varepsilon^{\prime}$ in mono-domain LATGS shows the feature just as that expected by the phenomenological Landau theory both above and below $T_{C}$ (Fig. 1), it is concluded that both the "plateau" region and the "kink" of $\varepsilon$ ' in TGS are closely related to DW's.

Within the temperature range of the "kink", there appears a $\varepsilon$ " peak $P_{3}$ (Fig. 1), and its position $T_{P}$ moves to higher $T$ with increasing $f$. Based upon standard relaxation theory, $2 \pi f \tau\left(\mathrm{T}_{\mathrm{P}}\right)=1$, i.e. $\tau(\mathrm{T} \mathrm{p})=1 / 2 \pi f$. Then the dependence of $\tau$ versus $T$ can be obtained as shown in Fig.2. Obviously, $\tau$ does not accord with Arrehnius relation $\tau=\tau_{0} \mathrm{e}^{\mathrm{U} / \mathrm{T}}$, but can be described by Vogel-Fulcher formula [9] in TGS (Insert of Fig.2).

$$
\tau=\tau_{0} e^{U /\left(T-T_{Y H}\right)}
$$


Based on the similarity of $\varepsilon^{\prime}$ and $\varepsilon$ " in TGS to those in KDP family, it would be concluded that there exist domain freezing in TGS, with freezing temperature $T_{f}$ about $32 \mathrm{~K}$.

$P_{1}$ and $P_{2}$ peak (Fig.1) have been discussed in Ref.[8]

\section{DISCUSSION}

Some striking feature related to domain freezing could be obtained by summarizing [Ref. [1-7] and present results] as the following:

(i) Domain freezing exists not only in KDP family, but also in TGS

(ii) During domain freezing, $\varepsilon$ ' decreases abruptly, looks like a "kink", and there appears a $\varepsilon$ " peak $\left(P_{3}\right)$.

Its relaxation time $\tau$ can be described by Vogel-Fulcher relation (Eq.1).

(iii) $T_{f}$ is dependent on defect density[10].

(iv) $\mathrm{T}_{\mathrm{f}}$ decreases with increasing sample thickness $\mathrm{d}$, and so on[6].

For Vogel-Fulcher relation indicates that $\tau \rightarrow \infty$ at finite temperature $T_{V F}$, it reflects the relaxation process related to phase transitions, such as the glass transition in polymers[11] and melting transition in solids [12]. Just based on this, Kuramoto [4] proposed that domain freezing is a glass transition of dipoles in DW's. Defects can pin DW's and the fact that $T_{f}$ is related to defects implies, a model of domain freezing should take the pinning effect of defects to DW's into account.

As pointed out by Bornarel [6], one must consider the dependence of $T_{f}$ versus $d$ when a domain freezing model is put forward. Experimental results shows the relation between $d$ and $D W$ density $N$ is $\mathrm{N} \sim \mathrm{d}^{-0.5}$ approximately, and it is confirmed by experimental observations[6] and theoretical calculations[8] that the interaction between DW's becomes stronger with increasing $N$. Therefore, the dependence of $T_{f}$ versus $\mathrm{d}$ implies that domain freezing is closely related to the interaction between DW's.

As mentioned above, $\tau$ of domain freezing accordance with Vogel-Fulcher relation reflects that there exists a transition at $\mathrm{T}_{\mathrm{VF}}$, i.e. an order appears on cooling or disappears on heating, and this order is closely related to the defect pinning to DW's and the interaction between DW's. An inspiring fact is that the collective-pinning of defects to fluxes has these features [13]. So, with the same manner as that of flux pinning, it is proposed that:

(i) At low temperature, there exist collective-pinning to DW's between random distribution defects i.e. the pinning of different defects in a certain spatial range in correlative; at the same time, due to the interaction between $D W$ 's, an effective pinning region $\Delta S$ like the boundle of vortexes [13] forms, i.e. the pinning in $\Delta S$ is correlative or local order $\Delta S$ appears. According to Ref. [13], the collective -pinning energy $\mathrm{U}_{\mathrm{CP}}$ is,

$$
U_{C P}=\sqrt{\left\langle U_{I P}(\vec{r}, \vec{u}) U_{I P}\left(\vec{r}^{\prime}, \vec{u}^{\prime}\right)\right\rangle}=U_{C P}\left(\vec{r}-\vec{r}^{\prime}, \vec{u}-\vec{u}^{\prime}\right)
$$

where $\vec{r}$ is the position of defects, $\vec{u}$ is the displacement vectors of DW's, and $U_{p i n}(\vec{r}, \vec{u})$ is individual pinning energy. One important effect of collective-pinning is that $U_{C P} \gg U_{\mathbb{P}}$.

(ii) With increasing $T$, collective-pinning becomes weaker or local order $\Delta S$ decreases due to thermal fluctuation, and at certain temperature $T_{f}$ this correlation will be destroyed totally or $\Delta S=0$. At the same time, $U_{C P}$ falls abruptly to $U_{\mathbb{R}}$, i.e. the dependence of pinning energy $U_{\text {pin }}$ versus $T$ near $T_{f}$ is,

$$
U_{p i n}=\left\{\begin{array} { l } 
{ U _ { C P } \text { for } T < T _ { f } \quad U _ { C P } > U _ { I P } } \\
{ U _ { I P } \text { for } T \geq T _ { f } }
\end{array} \rightarrow \left\{\begin{array}{l}
\infty \text { for } T<T_{f} \\
U_{I P} \text { for } T \geq T_{f}
\end{array}\right.\right.
$$

With continuous variation of $U_{\text {pin }}$ near $T_{f}$ being taken into account, $U_{\text {pin }}$ can be expanded approximately as the following,

$$
U_{p i n}= \begin{cases}\infty & \text { when } T<T_{f} \\ \frac{U}{T}-1 & \text { when } T \geq T_{f}\end{cases}
$$


Based upon Boltzmann statistic principle, the relation between the relaxation time $\tau$ during domain freezing and the pinning energy $U_{p i n}$ is,

$$
\tau= \begin{cases}\tau_{0} e^{\frac{U}{\left(T / T_{f}-1\right) T}} \stackrel{\text { near } T_{f}}{\longrightarrow} \tau_{0} e^{\frac{U}{T-T_{f}}} & \text { for } T>T_{f} \\ \rightarrow \infty & \text { for } T \leq T_{f}\end{cases}
$$

Eq.(5) is just Vogel-Fulcher relation with $T_{V F}$ equal to $T_{f}$, this means that the model proposed here can describe the relaxation process during domain freezing, and domain freezing is a process that a local pinning order $\Delta S$ appears due to collective-pinning effect, and the effective pinning energy increase abruptly during this process which leads to the relaxation time being accordance with Vogel-Fulcher relation.

Based on standard relaxation theory [14], it is obtained that the complex dielectric constant $\varepsilon_{D W}=\varepsilon_{D W}^{\prime}-i \varepsilon_{D W}^{\prime \prime}$ due to the relaxation of DW's during domain freezing is,

$$
\varepsilon=\varepsilon^{\prime}-i \varepsilon^{\prime \prime}= \begin{cases}\int_{0}^{\infty} \varepsilon_{D W}^{0} \frac{g(\tau)}{1+\omega^{2} \tau^{2}} \frac{d \tau}{\tau} & \text { for } T>T_{f} \\ \rightarrow 0 & \text { for } T \leq T_{f}\end{cases}
$$

where $\varepsilon_{D W}^{0}$ is the dielectric constant at low frequency limit. $\mathrm{g}(\tau)$ is the distribution function of $\tau$.

According to Eqs. (5)-(6), the calculated results of complex constant $\varepsilon_{D W}=\varepsilon_{D W}^{\prime}-i \varepsilon_{D W}^{\prime}$, due to the relaxation of DW's during domain freezing versus temperature with different frequency $f$, are the same as that shown in Figs. 1 and 2. Some key experimental results related to domain freezing, such as the sizeeffect of domain freezing, the two kinds of relaxation in low and high frequency ranges, respectively, etc. are also explained successfully (to be published elsewhere).

\section{Reference}

[1] Barkla H.M. and Finlayson D.M., Phil. Mag., 44(1953), 109

[2] Bornarel J., J. Appl. Phys. 43(1972), 3

[3] Fedosov V.N. and Sidorkin A.S., Sov. Phys. Solid, 19(1977), 1359

[4] Kuramoto K., J.Phys. Soc. Jpn., 56(1987), 1859

[5] Nakamura E. and Kuramoto K., J. Phys. Sol. Jpn, 51(1988), 2182

[6] Bornarel J. and Torche B., Ferroelectrics, 132(1992), 273

[7] Kubinec P., Fally M., Fuith A. et al., J. Phys.: Condens. Matter, 7(1995), 2205

[8] Huang Y.N., Wang Y.N. and Shen H.M., Phys. Rev. B 46(1992), 3290

[9] Vogel Z., Z. Phys. 22(1921), 645

Fulcher G.S., J. Am. Ceram. Soc., 8(1925), 339

[10] Kamysheva L.N. and Drozhdin S.N., Ferroelectrics 71(1987), 281

[11] McCrum N.G., <<Anelastic and Eielectric Effects in Polymer Solids〉>, John. Wiley \& Sons. INC., London, 1967

[12] Angell C.A., J. Non-Cryst. Solid 102(1988), 205

[13] Blatt G., Feigel'man, Larkin A.I., and Vinokur, Rev. Mod. Phys. 66, 1125(1994)

[14] Nowick A.S., Berry S., <<Anelastic Relaxation in Crystalline Solids $>$, Acdemic Press, INC, New York and London, 1972 